\title{
Optimisation mathématique des séries temporelles. Nettoyage des séries financières des effets calendriers et saisonniers -Cas du marché financier marocain-
}

\author{
Mathematical optimization of time series.
}

Cleaning financial series of calendar and seasonal effects -Moroccan financial market-

\author{
Mustapha Bouchekourte ${ }^{1}$, Norelislam El Hami ${ }^{2}$ \\ ${ }^{1}$ Laboratoire Sciences de Gestion, Univérsité Hassan II - Casablanca, Maroc, bouchekourte@depf.finances.gov.ma \\ ${ }^{2}$ Laboratoire Génie des Systèmes (LGS), ENSA-Kénitra, Université Ibn Tofail, Maroc, norelislam@outlook.com
}

RÉSUMÉ. La construction du calendrier national permet l'optimisation et la production des séries corrigées des variations saisonnières, et donc d'améliorer significativement la qualité des modèles utilisés pour obtenir des indicateurs fiables, lisibles, interprétables et comparables. La décomposition des séries permet d'améliorer la qualité des régressions en isolant les effets irréguliers, saisonniers et de calendriers qui peuvent biaiser les relations modélisables. Nous allons analyser les séries de variables explorées en utilisant le logiciel Demetra+ [1] pour mieux affiner nos estimations par la suite. L'analyse exploratoire des données utilisées devrait permettre de résumer la distribution de chaque série et les relations entre les variables dont les caractéristiques pourraient exiger des transformations de mesure et d'unité ou des recodages. Les outliers par exemple sont susceptibles d'influencer les résultats d'un modèle statistique. Ce traitement permettra de capter les valeurs influentes avant de modéliser les corrélations entre les variables faisant l'objet d'une estimation.

ABSTRACT. The construction of the national calendar allows the optimization and the production of the seasonally adjusted series, thus significantly improving the quality of the models used to obtain reliable, legible, and interpretable indicators. Series decomposition improves the quality of regressions by isolating irregular, seasonal, and time-sensitive effects that can bias the modeled relationships. We will analyze the series of variables explored using the Demetra + software [1] to better refine our estimates. The exploratory analysis of the data used should make it possible to summarize the distribution of each series and the relationships between variables whose characteristics might require measurement and unit transformations (or recoding). Outliers, for example, are likely to influence the results of a statistical model. This treatment will capture the influential values before modeling the correlations between the variables being estimated.

MOTS-CLÉS. Effets irréguliers et calendriers, qualité des regressions, optimisation.

KEYWORDS. Irregular effects and schedules, quality of regressions, optimization.

\section{Introduction}

Pour analyser la structure et les indicateurs de microstructure de la bourse de Casablanca, dont la fréquence quotidienne peut avoir un caractère saisonnier nous procéderons par la suite, dans la deuxième section, à la sélection et au traitement des variables et des séries qui feront l'objet de notre exploration économétrique. Nous allons extraire, en plus des effets saisonniers, les effets du calendrier en introduisant des régresseurs propres au calendrier marocain [2] pour améliorer l'utilisation des séries économiques et financières influencées par la fréquence mobile des jours fériés (fêtes religieuses et nationales). Ce traitement améliorera la qualité et la significativité des tests économétriques.

\section{La désaisonnalisation et la correction calendaire des séries}

La désaisonnalisation seule des données financières (de microstructure) ne permet pas de corriger correctement les séries chronologiques, puisque celles-ci sont aussi rythmées aux spécificités du 
calendrier. La correction des effets de calendrier doit être, ainsi, menée parallèlement à toute opération de désaisonnalisation, afin d'identifier correctement les fluctuations conjoncturelles. Les effets de calendrier ne se confondent pas totalement avec ceux liés à la saisonnalité (pure). Si cette dernière traite des phénomènes ayant, par définition, une date grégorienne fixe, les premiers englobent, en outre, les effets liés à la nature des jours et aux événements dont les dates sont mobiles. C'est ainsi que le traitement calendaire se focalise sur deux types de variations. Le premier porte sur celles relatives au nombre de jours ouvrés ou ouvrables d'un mois à l'autre (ou toute période infra-annuelle). Plus spécifiquement, il s'intéresse aux modifications dues à la composition en jours de la semaine. Le deuxième type regroupe, quant à lui, les jours fériés par référence aux fêtes civiles ainsi que les fêtes mobiles, telles que le ramadan ou aïd Adha. Cette étape permettrait d'avoir des séries corrigées [3] des variations saisonnières et des effets de calendrier et d'améliorer significativement la qualité de la production tant au niveau de l'observation statistique qu'au niveau de la prévision financière.

\section{Le modèle de base pour les jours ouvrables dans le calendrier grégorien}

Contrairement aux anciennes approches, fondées sur l'idée de proportionnalité, la correction des effets du calendrier s'effectue désormais à travers une démarche économétrique, basée sur la construction des variables exogènes (dénommées régresseurs ${ }^{1}$ ). Elle permet ainsi de tester leur existence et d'estimer statistiquement leur impact. Il faut dire que le caractère particulier du calendrier, essentiellement sa fréquence d'occurrence assez différente de celle des observations, empêche tout recours aux moyennes mobiles et aux modèles ARIMA pour capter ces phénomènes. Le modèle de base développé dans la littérature consiste à décomposer la série selon les jours de la semaine :

$$
X_{t}=\sum_{i=1}^{7} \alpha_{i} N_{i t}+\varepsilon_{t}
$$

Où : Xt : série brute,

Nit : Nombre de lundis (i=1), Mardis (i=2) ... à la date $\mathrm{t}$ (mois, trimestre)

Et: un terme d'erreur qui suit un modèle ARIMA (ou SARIMA).

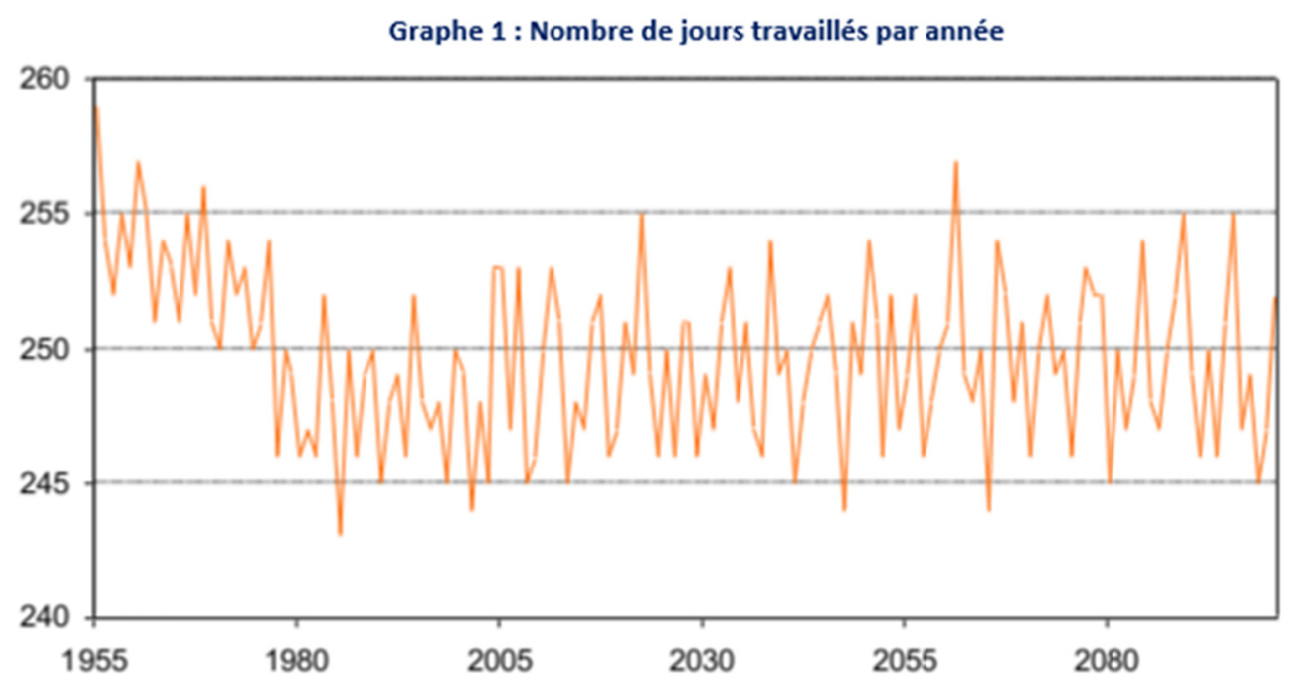

\footnotetext{
${ }^{1}$ Le régresseur utilisé dans notre travail de recherche est élaboré par le Haut-Commissariat au Plan qui l'utilise pour améliorer la qualité des études économiques et établir des analyses économétriques moins biaisées surtout que les analyses de conjoncture se basent quasi-exclusivement sur le suivi des évolutions annuelles, trimestrielles ou mensuelles d'un ensemble d'agrégats socioéconomiques. 6 Ainsi pour exemple, la fête de "Aid El-Fitr » est survenue deux fois pendant les années 1968 et 2000. Ce phénomène se répétera durant les années 2033, 2065 et 2098. 
Il peut arriver parfois qu'une fête donne lieu à trois jours fériés dans l'année (cas de Aid Adha dans les années 2006 et 2007). En dehors de ce caractère chômé de ces fêtes, c"est leur impact social qui peut vraisemblablement être le plus à l'origine de ces effets annuels. Dans le même sens, le nombre de jours jeûnés (du mois de Ramadan) oscille lui aussi nettement d'une l'année à l'autre. Avec un minimum de 29 jours dans l'année, ce nombre peut atteindre un maximum de 41 jours.

Graphe 2 : Nombre de jours fériés et jeûnés par années

ADHA

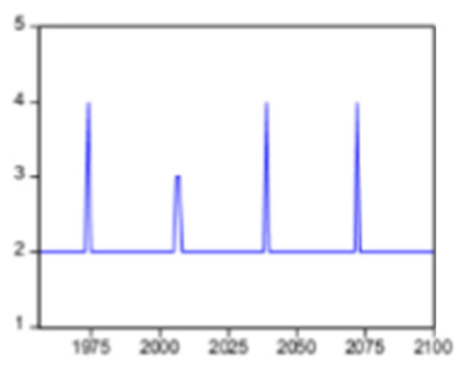

ATrE

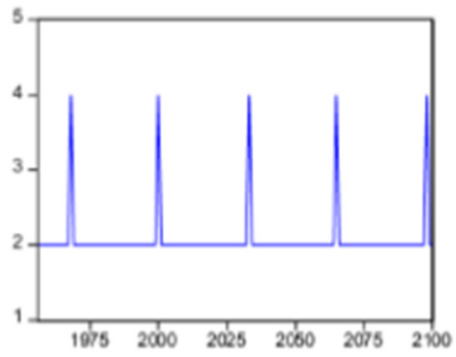

RAMADAN

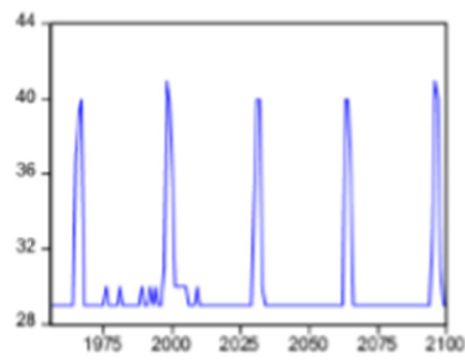

La méthode d'ajustement saisonnier X12-ARIMA [4] a été appliquée sur des séries mensuelles des indicateurs relatifs au marché boursier (et au marché des capitaux en général) couvrant la période allant de janvier 2004 à décembre 2014. Cette méthode est fondée sur le lissage des séries à travers les moyennes mobiles. Elle est implémentée dans le logiciel "DEMETRA+" dêveloppé par EUROSTAT [5].

\section{Résultats de l'introduction du régresseur du calendrier marocain}

Pour corriger les effets de calendrier susceptibles de perturber la tendance fondamentale des séries traitées, il a été décidé d'introduire les régresseurs associés au calendrier national (jours ouvrables du mois, fêtes religieuses, Ramadan...etc.) pour qu'ils soient corrigés au niveau d'une phase préalable à la décomposition de la série, ce qui permet d'améliorer la qualité de l'ajustement saisonnier.

\begin{tabular}{c|c|c|c|c|c|c|c|c|}
\multicolumn{1}{c|}{ Variable } & Part & RI & Volmasi & Ctm13 & Volmadex & Vtmc & Ncmc & Ctm10 \\
\hline $\begin{array}{c}\text { Trading } \\
\text { days } \\
\text { effects }\end{array}$ & $\begin{array}{c}\text { No trading } \\
\text { days } \\
\text { effects }\end{array}$ & $\begin{array}{c}\text { No trading } \\
\text { days effects }\end{array}$ & $\begin{array}{c}\text { No trading } \\
\text { days effects }\end{array}$ & $\begin{array}{c}\text { No trading } \\
\text { days effects }\end{array}$ & 1 variable & 1 variable & 1 variable & $\begin{array}{c}2 \\
\text { variables }\end{array}$ \\
\hline Summary & Good & Uncertain & Uncertain & Good & Uncertain & Uncertain & Good & Good \\
\hline Normality & $\begin{array}{c}\text { Uncertain } \\
(0,048)\end{array}$ & Bad $(0,000)$ & Good $(0,474)$ & $\begin{array}{c}\text { Uncertain } \\
(0,021)\end{array}$ & Good $(0,206)$ & Bad $(0,000)$ & $\begin{array}{c}\text { Good } \\
(0,712)\end{array}$ & $\begin{array}{c}\text { Bad } \\
(0,000)\end{array}$ \\
\hline $\begin{array}{c}\text { Residual } \\
\text { seasonality }\end{array}$ & $\begin{array}{c}\text { Good } \\
(0,974)\end{array}$ & $\begin{array}{c}\text { Good } \\
(0,966)\end{array}$ & Good $(0,928)$ & $\begin{array}{c}\text { Good } \\
(1,000)\end{array}$ & Good $(0,994)$ & $\begin{array}{c}\text { Good } \\
(1,000)\end{array}$ & $\begin{array}{c}\text { Good } \\
(1,000)\end{array}$ & $\begin{array}{c}\text { Good } \\
(1,000)\end{array}$ \\
\hline
\end{tabular}

\begin{tabular}{|c|c|c|c|c|c|c|c|c|}
\hline Variable & Ctm52s & In $\mathrm{m} 3$ & Ln $\mathrm{m} 1$ & $\begin{array}{c}\text { Opcrm } \\
\text { monétaire }\end{array}$ & Omlt & $\begin{array}{l}\text { Opcrm } \\
\text { actions }\end{array}$ & Masi & Ncmb \\
\hline $\begin{array}{c}\text { Trading } \\
\text { days } \\
\text { effects }\end{array}$ & $\begin{array}{l}\text { No trading } \\
\text { days effects }\end{array}$ & 1 variable & 1 variable & 2 variables & 2 variables & $\begin{array}{l}\text { No trading } \\
\text { days effects }\end{array}$ & $\begin{array}{l}\text { No trading } \\
\text { days effects }\end{array}$ & $\begin{array}{c}2 \\
\text { variables }\end{array}$ \\
\hline Summary & Good & Good & Uncertain & Good & Uncertain & Uncertain & Good & Uncertain \\
\hline Normality & $\begin{array}{c}\text { Uncertain } \\
(0,064)\end{array}$ & $\begin{array}{l}\text { Good } \\
(0,992)\end{array}$ & $\begin{array}{c}\text { Uncertain } \\
(0,014)\end{array}$ & Good $(0,846)$ & $\operatorname{Bad}(0,000)$ & Bad $(0,000)$ & $\begin{array}{c}\text { Uncertain } \\
(0,019)\end{array}$ & $\begin{array}{c}\text { Bad } \\
(0,000)\end{array}$ \\
\hline $\begin{array}{c}\text { Residual } \\
\text { seasonality }\end{array}$ & Good $(0,999)$ & $\begin{array}{c}\text { Good } \\
(1,000)\end{array}$ & Good $(1,000)$ & Good $(0,994)$ & Good $(0,992)$ & Good $(0,996)$ & Good $(0,996)$ & $\begin{array}{c}\text { Good } \\
(0,972)\end{array}$ \\
\hline
\end{tabular}

D'après le tableau sur les niveaux de qualité possibles pour chacun des diagnostics, n'ont pas tous le même niveau de gravité. Seuls les tests de respect des contraintes (basic checks) peuvent mettre en erreur la série.

Les résidus du modèle sont utiles pour vérifier la qualité de l'ajustement saisonnier [6]. Les résidus doivent être indépendants et aléatoires, et donc suivre la distribution normale. Ils ne doivent pas 
contenir des informations, telles que la saisonnalité et ils devraient être répartis selon une distribution normale.

Le commentaire sur la qualité globale de la désaisonnalisation est confectionné à partir des commentaires sur les différentes catégories de statistiques. Ce commentaire indique si oui ou non la désaisonnalisation est acceptable. La qualité globale de traitement de la série fait l'objet d'un commentaire synthétique.

\begin{tabular}{|c|c|c|c|}
\hline \multicolumn{4}{|c|}{ Effets calendriers détectés } \\
\hline Variables & Effets validés & F-Test global & P-Value \\
\hline Part & LeapY, Raselam et Wd & $F=5,0431$ & {$[=0,0001]$} \\
\hline $\mathbf{R L}$ & Aucun effet signifiant & $F=0,6421$ & {$[=0,6963]$} \\
\hline VolMASI & Aucun effet signifiant & $\mathrm{F}=0,5632$ & {$[=0,7587]$} \\
\hline CTMS52S & Lundi et Mardi & $\mathrm{F}=2,4680$ & {$[=0,0281]$} \\
\hline VolMadex & Fitr & $F=3,6639$ & {$[=0,0023]$} \\
\hline VT & Adha & $\mathrm{F}=10,6638$ & {$[=0,0000]$} \\
\hline NCMC & Lundi & $F=10,1874$ & {$[=0,0000]$} \\
\hline NCMB & Mardi \& Mercredi & $\mathrm{F}=6,1127$ & {$[=0,0000]$} \\
\hline CTMS10 & Mardi \& Mercredi & $F=2,4485$ & {$[=0,0290]$} \\
\hline CTMS52S & Aucun effet signifiant & $F=0,3938$ & {$[=0,8816]$} \\
\hline $\ln (\mathrm{M} 3)$ & Adha & $\mathrm{F}=3,6151$ & {$[=0,0025]$} \\
\hline Ln(M1) & Adha & $F=4,7827$ & {$[=0,0002]$} \\
\hline OPCVM monétaires & Lundi \& Vendredi & $\mathrm{F}=3,0630$ & {$[=0,0081]$} \\
\hline OMLT & Aucun effet signifiant & $F=0,8247$ & {$[=0,5533]$} \\
\hline OPCVM actions & Aucun effet signifiant & $\mathrm{F}=0,7991$ & {$[=0,5728]$} \\
\hline MASI & Aucun effet signifiant & $\mathrm{F}=0,9721$ & {$[=0,4479]$} \\
\hline
\end{tabular}

Calculs auteur pars Demetra+

La série désaisonnalisée et sans effet calendrier est égale à la composante saisonnière plus la composante irrégulière plus les effets des autres régresseurs sur la série brute.

Il est possible de connaître pour chaque série, la note obtenue selon les différents axes de qualité, à savoir :

- Qualité des points aberrants ;

- Qualité des résidus du modèle ARIMA;

- Qualité de décomposition;

- Absence d'effets résiduels ;

- Qualité des révisions.

Différentes possibilités en termes de spécifications sont testées [7]. En particulier, une désaisonnalisation automatique de toutes les séries avec une nouvelle recherche des points aberrants sur toute la période est pratiquée. Un bilan de la qualité est édité pour chacune des versions confectionnées. Les qualités des désaisonnalisations effectuées avec les différentes spécifications suivant les différentes catégories de diagnostics sont comparées afin de déterminer le meilleur modèle pour chaque série.

Le résumé final de la qualité est élaboré. Il s'agit de pointer les séries contribuant le plus à la dégradation de la qualité (analyse descendante). La méthode X-119 applique les moyennes mobiles [8] pour arriver à la tendance-cycle et à la composante saisonnière. La série brute est divisée par la composante saisonnière afin d'obtenir la série désaisonnalisée en cas de décomposition multiplicative. Dans le cas d'une composition d'additive, la composante saisonnière est déduite de la série brute. La batterie d'indicateurs des sorties présente les composantes, saisonnière, irrégulière, de tendance, ajustée et finale en plus d'une série prévisionnelle des séries des variables traitées. Le niveau d'ajustement de ces séries correspond, sur le graphique, au degré de décalage entre la série brute et la série ajustée pour chaque variable. 


\section{Résultats}

La désaisonnalisation des séries utilisées a permis de relever les constatations suivantes :

- Les indicateurs relatifs à la bourse de Casablanca sont plus saisonniers que ceux relatifs à la courbe des taux et aux autres aléas du marché des capitaux ;

- L'effet Ramadan est généralement négligeable pour la totalité des variables, toutefois, il est perceptible sur des composantes spécifiques comme le volume des transactions et le nombre de titre échangés ;

- Les fluctuations liées aux fêtes religieuses sont moins importantes que celles des liées aux jours ouvrables ;

- L'effet des jours ouvrables s'avère signifiant au niveau de la majorité des variables surtout pour quelques jours de la semaine (comme Mardi et Jeudi).

En plus de ceux liés à la saisonnalité «pure», sont considérés comme des facteurs techniques. Pour dégager les fluctuations de fonds, inhérentes au comportement économique des agents, il est nécessaire de faire passer les séries chronologiques aux filtres de désaisonnalisation et de correction calendaire.

\section{Bibliographie}

[1] Necmettin Alpay KOÇAK, « On analysis of German Industrial Production with Demetra+ », Turkish Statistical Institute.

[2] Bulletin Officiel N²406 pages1986 et 2539 (années 1958 et 1961)

- B.O. N³927 page 55 (année1988).

- B.O. N4159 page 332 (année 1992).

- B.O. N4205 page 223 (année 1993).

- B.O. N4796 pages 336, 345 (année 2000).

[3] Mustapha BOUCHEKOURTE, «Allocations d'actifs et leurs impacts sur le marché des capitaux) - Cas de la bourse de Casablanca -

[4] Sylwia Grudkowska, «Demetra+ User Manual», National Bank of Polan

[5] Site internet d'Eurostat : https://ec.europa.eu/eurostat/sa-elearning/.

[6] Greene W. H., 2007, Econometric analysis. 6 ed. Upper Saddle River: Prentice Hall.

[7] Sylwia Grudkowska, National Bank of Poland,"Demetra+ User Manual", Octobre 2011.

[8] Necmettin Alpay KOÇAK, " On analysis of German Industrial Production with Demetra+ », Turkish Statistical Institute. 